\title{
Characterizing temporal coherence of visible synchrotron radiation with heterodyne near field speckles
}

\author{
M. Siano, B. Paroli, and M. A. C. Potenza \\ Dipartimento di Fisica, Università degli Studi di Milano and INFN Sezione di Milano, \\ via G. Celoria, 16, 20133 Milano, Italy \\ U. Iriso, A. A. Nosych, and L. Torino \\ ALBA-CELLS Synchrotron Radiation Facility, \\ Carrer de la Llum 2-26, 08290 Cerdanyola del Vallès (Barcelona), Spain
}

S. Mazzoni, G. Trad, and A. N. Goldblatt

CERN, CH-1211 Geneva, Switzerland

(Received 6 July 2017; published 21 November 2017)

\begin{abstract}
In this paper we extensively describe the heterodyne near field speckle method (HNFS) to characterize both spatial and temporal coherence of synchrotron radiation (SR). The method relies on Fourier analysis of near field speckles generated by scattering from nanoparticles suspended in a liquid. A criterion based on master curves of power spectra is introduced and validated by measurements on the visible light produced by the ALBA bending dipole. While spatial coherence measurements with HNFS have been reported, we present for the first time measurements of the temporal coherence of SR wavefronts with the HNFS method both for narrowband and white light beams. In the former case, using a band-pass filter, a coherence time of $40 \pm 10 \mathrm{fs}$ is measured, in good agreement with the expected value of $43 \mathrm{fs}$ for the filter inverse linewidth. Moreover, by exploiting the self-reference scheme of the technique, we show that coherence areas propagate carrying nonvanishing curvature. In the latter case, the measured coherence time of the incident SR without any monochromator is $1.6 \pm 0.4 \mathrm{fs}$, corresponding to a bandwidth of $240 \mathrm{~nm}$ at a peak wavelength of $350 \mathrm{~nm}$. Exploiting the Wiener-Kintchine theorem, we also retrieve the SR power spectral density at the sample position from the measured temporal coherence function. Results are in good agreement with the measurements performed using a standard spectrometer, yielding a coherence time of 1.4 fs.
\end{abstract}

DOI: 10.1103/PhysRevAccelBeams.20.110702

\section{INTRODUCTION}

Synchrotron radiation (SR) is nowadays an outstanding research tool thanks to the development of third-generation light sources delivering photon beams with high brightness and high degree of coherence [1]. Many experimental techniques exploiting coherent radiation are routinely implemented at large-scale facilities, e.g., scattering, photon correlation spectroscopy and phase contrast imaging [2]. In this framework, characterization of spatial and temporal coherence properties of the radiation at the sample position is fundamental for proper planning of the experiments and for unbiased data reduction. Measurements of spatial coherence of SR also provide a noninvasive diagnostics tool for the particle beam itself, yielding information on the electron beam transverse distribution

*mirko.siano@unimi.it

Published by the American Physical Society under the terms of the Creative Commons Attribution 4.0 International license. Further distribution of this work must maintain attribution to the author(s) and the published article's title, journal citation, and DOI.
[3,4]. More recently, the X-ray and gamma-ray betatron radiation from laser-plasma acceleration has been studied both theoretically and experimentally [5-7] to diagnose the particle beam inside the plasma through the emitted spectral density [7] and related temporal coherence properties $[6,8]$. Consequently, accurate characterization and control of spatial and temporal coherence properties of the SR is an increasingly challenging demand in modern synchrotron and laser-plasma based light sources.

Widespread techniques for the characterization of spatial coherence of SR are based on interference from a double aperture or on Fresnel diffraction by a slit $[9,10]$. Several solutions have been adopted to perform two-dimensional (2-D) coherence measurements and to extend such techniques to $\mathrm{x}$-rays [11-15]. They typically require moving and orienting probing devices, careful engineering of masks and gratings, fine alignment of the optical components and implementation of dedicated x-ray optics. The same drawbacks arise for temporal coherence measurements, usually performed with amplitude-division interferometers based on the Michelson scheme [16,17].

Alaimo et al. [18] recently proposed a novel technique based on Fourier analysis of heterodyne near field speckles 
(HNFS) obtained with a scheme that is reminescent of the in-line Gabor holography [19]. It exploits the staggered interference between the spherical waves scattered by nanoparticles in a colloidal suspension and the intense transmitted beam to measure the full 2-D transverse coherence function of SR without any a priori assumption [20]. Remarkably, the experimental setup is minimal and free of any severe alignment requirement. Moreover, the method can be easily scaled to any wavelength. For x-rays, it can be operated without any dedicated optics $[18,21]$. Furthermore, it has been recently shown [22] with tabletop optical sources that the same technique allows careful measurements of temporal coherence thanks to small optical path differences between the scattered and the transmitted wavefronts.

Here we rigorously discuss how to apply the HNFS technique to the characterization of the full spatio-temporal coherence properties of SR and we show for the first time results regarding temporal coherence properties of the visible part of synchrotron light produced by a bending dipole. Moreover, we investigate the possibility of measuring the wavefront curvature by scanning different sample-detector distances.

The presence of optical components along the beamline prior to the detection point might introduce some changes in the SR spectrum and related coherence properties. In coherence-based experiments this is of no concern as one is interested in the coherence properties of the radiation at the sample position. Conversely, coherence-based electron beam diagnostics would demand the extraction line to be absent or at least its spectral response to be characterized in order to retrieve properties of the electron bunch from the measured coherence functions. Either way, knowledge of the coherence properties of SR at some position along the beamline is of fundamental importance and it represents the aim of our work. The reader should be aware that for diagnostics purposes a characterization of the spectral response of the extraction line is also demanded.

The paper is organized as follows: in Sec. II we provide an introduction on the HNFS diagnostics technique; in Sec. III we describe the ALBA facility and experimental setup installed on the Xanadu beamline; Sections IV and V show experimental results regarding temporal coherence of narrowband and white SR beams, respectively, as well as the effects of wavefront curvature on the technique; finally, we collect our conclusions in Sec. VI.

\section{THEORY OF THE HETERODYNE NEAR FIELD SPECKLE METHOD}

The HNFS method $[23,24]$ is a self-referencing technique based on the interference between the weak field scattered by spherical nanoparticles suspended in a liquid and the strong transmitted radiation (heterodyne condition). Similarly to in-line holography [19], this condition preserves phase information and it therefore allows to probe coherence properties of the incoming beam. The continuous Brownian motion of the particles guarantees a complete statistical renewal of the sample in times that can be easily set large enough to not affect data but short enough with respect to subsequent measurements [24].

The typical experimental layout of an HNFS experiment is sketched in Fig. 1. A colloidal particle illuminated by the impinging SR scatters a weak, almost perfect spherical wave which is heterodyned by the strong transmitted wavefront. The superposition of the two fields at a distance $z$ downstream the particle generates instantaneous interference fringes of the form

$$
I_{i}^{\text {inst }}(x, y, z)=\left|E_{0}(x, y, z)+\frac{|S(0)|}{k r} E_{0}\left(x_{i}, y_{i}, 0\right) e^{i k r}\right|^{2},
$$

where $E_{0}$ is the electric field of the incoming beam and $S(0)$ is the forward scattering amplitude of the particle $i$. The coordinates $(x, y)$ denote the transverse position on the detection plane at a distance $z$ from the cuvette and $\left(x_{i}, y_{i}\right)$ correspond to the coordinates of the particle $i$ inside the cuvette. The distance $r$ is defined as $r \approx$ $z+\left[\left(x-x_{i}\right)^{2}+\left(y-y_{i}\right)^{2}\right] /(2 z)$ in paraxial approximation and $k=2 \pi / \lambda$ is the radiation wave number, with $\lambda$ the radiation wavelength.

In heterodyne conditions, the term $\mid S(0) E_{0}\left(x_{i}, y_{i}, 0\right) e^{i k r} /$ $\left.(k r)\right|^{2}$ can be neglected and Eq. (1) becomes simply

$$
\begin{aligned}
I_{i}^{\text {inst }}(x, y, z)= & I_{0}(x, y, z) \\
& +\frac{2|S(0)|}{k r} \Re e\left\{E_{0}(x, y, z) E_{0}^{*}(x, y, 0) e^{-i k r}\right\},
\end{aligned}
$$

where $I_{0}(x, y, z)=\left|E_{0}(x, y, z)\right|^{2}$ and $\Re e$ denotes the real part of complex numbers.

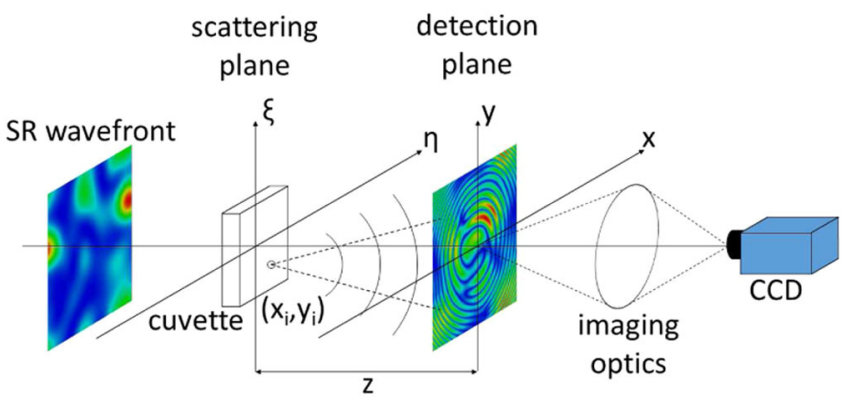

FIG. 1. Typical HNFS experimental setup. A SR wavefront impinges onto a quartz cuvette containing many scattering particles suspended in a liquid at random positions $\left(x_{i}, y_{i}\right)$. The interference between the scattered spherical waves and the transmitted radiation is observed across the detection plane $(x, y)$ at a distance $z$ from the scattering plane $(\eta, \xi)$ by means of a CCD camera (the case of a single-particle is shown here for illustrative purposes). Magnifying imaging optics may be used, depending on the spatial resolution to be achieved. 
Next we show how to retrieve the spatial and temporal coherence of SR wavefronts with the HNFS technique.

\section{A. Spatial coherence}

Typically, SR wavefronts by charged particle beams of finite emittance can be described as a disordered ensemble of many patches where the phase of the electric field $E_{0}$ is constant, in a way similar to the speckled intensity distribution characterizing diffuse laser light [25]. Due to the finite bandwidth of SR, the coherence patches are short-lived, changing in shape and position with a characteristic time $\tau_{\mathrm{ch}}$ typically ranging between $10^{-17} \mathrm{~s}$ and $10^{-14} \mathrm{~s}[26,27]$. Detection of the interference fringes described by Eq. (2) with an exposure time shorter than $\tau_{\mathrm{ch}}$ would probe such coherence areas by means of the features of distorted fringes, as depicted in Fig. 2(a). Actually, only temporal averages of a large number of ensemble realizations are accessible. Statistical properties of SR wavefronts can then be described by introducing the complex coherence factor [CCF or $\mu(\Delta x, \Delta y)][27]$

$$
\begin{aligned}
& \mu(\Delta x, \Delta y)=\frac{\left\langle E(x, y) E^{*}(x+\Delta x, y+\Delta y)\right\rangle}{\sqrt{I_{1} I_{2}}} \\
& I_{1}=\left\langle E(x, y) E^{*}(x, y)\right\rangle \\
& I_{2}=\left\langle E(x+\Delta x, y+\Delta y) E^{*}(x+\Delta x, y+\Delta y)\right\rangle,
\end{aligned}
$$

where $\langle\cdot\rangle$ denotes ensemble averages over many electron bunches and $\Delta x$ and $\Delta y$ are the deviations from $(x, y)$. Furthermore, with the assumptions of uniform intensity $\left(I_{1}=I_{2}\right)$, of the paraxial approximation and of plane wave propagation for the coherence areas

$$
E_{0}(x, y, z)=E_{0}(x, y, z=0) e^{i k z}
$$

we can rewrite Eq. (2) as

$$
I_{i}(x, y, z)=\left\langle I_{i}^{\text {inst }}(x, y, z)\right\rangle=\left\langle I_{0}(x, y, 0)\right\rangle \times\left\{1+\frac{2|S(0)|}{k r}\left|\mu\left(\Delta x_{i}, \Delta y_{i}\right)\right| \cos \left[k \frac{\Delta x_{i}^{2}+\Delta y_{i}^{2}}{2 z}\right]\right\},
$$

where $\Delta x_{i}=x-x_{i}, \Delta y_{i}=y-y_{i}$ and the phase of $\mu\left(\Delta x_{i}, \Delta y_{i}\right)$ has been put to zero without loss of generality since it just causes a phase shift of the interference fringes.

Equation (5) describes circularly symmetric interference fringes whose visibility $V=\left\{I_{i}^{\max }-I_{i}^{\min }\right\} /\left\{I_{i}^{\max }+I_{i}^{\min }\right\}$ is reduced according to the 2-D CCF of the incident SR [27,28], as shown in Fig. 2(b). Here $I_{i}^{\max }=1+\{2|S(0)| /(k r)\}$ $\left|\mu\left(\Delta x_{i}, \Delta y_{i}\right)\right|$ and $I_{i}^{\min }=1-\{2|S(0)| /(k r)\}\left|\mu\left(\Delta x_{i}, \Delta y_{i}\right)\right|$.

Using a colloidal suspension, the interference between the synchrotron radiation and the $N$ spherical waves scattered by the suspended particles results in the superposition of many single-particle interferograms as in
Fig. 2(c), which eventually provides a stochastic heterodyne speckle field for large $N$, as shown in Fig. 2(d):

$$
I_{\mathrm{tot}}=\sum_{i=1}^{N} I_{i}(x, y, z)=\tilde{I}(x, y, z) \otimes \sum_{i=1}^{N} \delta\left(x_{i}, y_{i}\right),
$$

where $\tilde{I}(x, y, z)$ is Eq. (5) for a particle at position $(0,0)$, $\delta\left(x_{i}, y_{i}\right)$ describes a pointlike scatterer at position $\left(x_{i}, y_{i}\right)$ and $\otimes$ denotes convolution.

Despite fringe visibility is not directly measurable, spatial coherence is probed by means of Fourier analysis as the 2-D power spectrum of heterodyne speckles $I(\vec{q})$ is directly related to the single-particle interferogram:
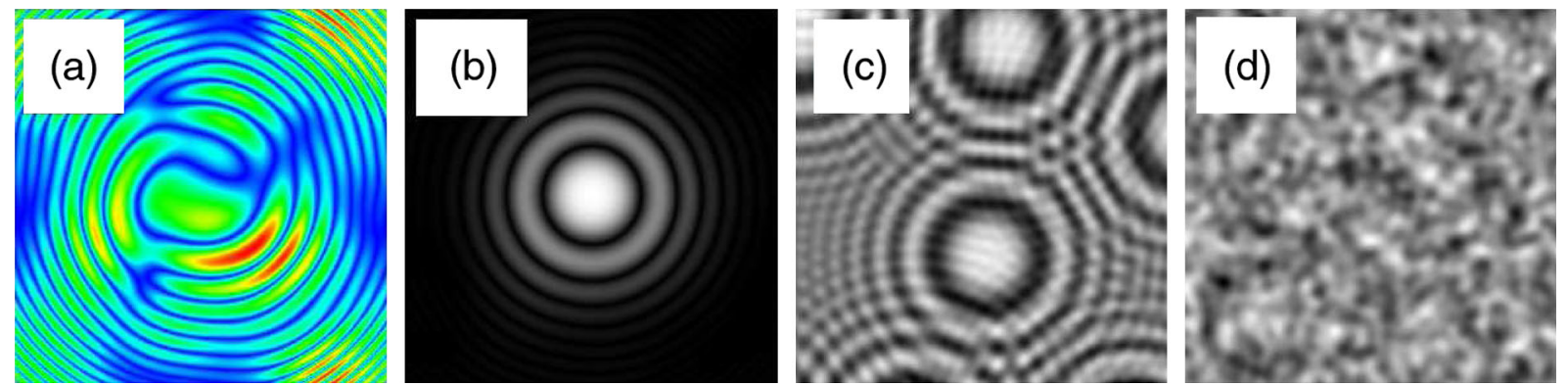

FIG. 2. Fundamentals of the heterodyne near field speckle technique. Simulation parameters are $\lambda=440 \mathrm{~nm}$ and $z=2 \mathrm{~mm}$. A 2-D Gaussian coherence factor of the form $\exp \left(-\left[x^{2}+y^{2}\right] / \sigma_{\text {coh }}^{2}\right)$ has been used, with $\sigma_{\text {coh }}=60 \mu \mathrm{m}$. (a) Time-resolved interference fringes generated by the superposition of the spherical wave scattered by one colloidal particle and the transmitted partially coherent SR wavefront. (b) Time-integrated single-particle interferogram. (c) Sum of $N=10$ heterodyne interferograms: interference fringes are still visible and may allow coherence measurements through their visibility. (d) Speckle field from $N=10000$ colloidal particles: interference fringes are no more visible but coherence properties are still retrievable in Fourier space. 


$$
\begin{aligned}
I(\vec{q}) & =\left|\mathcal{F}\left\{I_{\text {tot }}(x, y, z)\right\}(\vec{q})\right|^{2} \\
& =\left|\mathcal{F}\left\{\tilde{I}(x, y, z) \otimes \sum_{i=1}^{N} \delta\left(x_{i}, y_{i}\right)\right\}(\vec{q})\right|^{2} \\
& =|\mathcal{F}\{\tilde{I}(x, y, z)\}(\vec{q})|^{2} \times\left|\mathcal{F}\left\{\sum_{i=1}^{N} \delta\left(x_{i}, y_{i}\right)\right\}(\vec{q})\right|^{2},
\end{aligned}
$$

where $\vec{q}=\left(q_{x}, q_{y}\right)$ is the 2-D Fourier wave vector and $\mathcal{F}$ denotes the 2-D Fourier transform.

Replacing point-like scatterers with spherical spheres of finite extent and performing a further convolution of Eq. (6) with the response function of the detection optics, Eq. (7) can be generalized to $[18,20]$

$$
I(\vec{q})=S(\vec{q}) T(\vec{q}) C(\vec{q}) H(\vec{q})+P(\vec{q}),
$$

where $S(\vec{q})$ is the particle form factor of the scattering spheres [29], $T(\vec{q})=\sin ^{2}\left\{z|\vec{q}|^{2} /(2 k)\right\}$ is known as the Talbot transfer function and it describes the power spectrum oscillations visible in Fig. 3(a) and 3(b) and related to the oscillating term in the single-particle interferogram described by Eq. (5), $C(\vec{q})=|\mu(\vec{q})|^{2}$ is the squared modulus of the radiation CCF, $H(\vec{q})$ is the response function of the detection optics and $P(\vec{q})$ accounts for spurious contribution in power spectra, mainly shotnoise and readout noise of the detection system.

The information on the coherence is carried by the envelope of the Talbot oscillations, the term $C(\vec{q}) T(\vec{q})$ oscillating between 0 and $C(\vec{q})$. To obtain this curve, one has to consider the other components of Eq. (8). In particular, the shotnoise contribution $P(\vec{q})$ can be measured without the colloidal suspension, the particle form factor $S(\vec{q})$ can be calculated according to the Mie theory or measured with scattering experiments and the instrumental
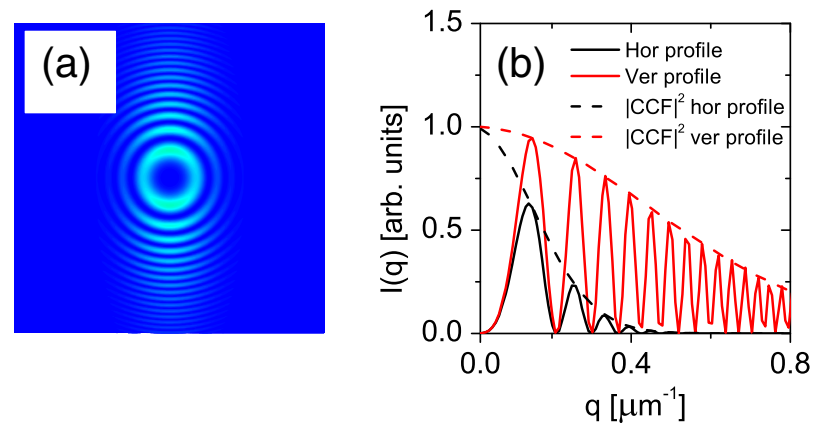

FIG. 3. (a) Simulated 2-D power spectrum $I(\vec{q})$ of heterodyne speckles and (b) radial profiles along the horizontal (solid black line) and vertical direction (solid red line), showing Talbot oscillations enveloped by the radiation CCF. Here $q=|\vec{q}|$. Simulation parameters are $\lambda=440 \mathrm{~nm}$ and $z=2 \mathrm{~mm}$. A 2-D Gaussian coherence factor of the form $\exp \left(-x^{2} / \sigma_{\text {coh.x }}^{2}\right)$ $\exp \left(-y^{2} / \sigma_{\text {coh,y }}^{2}\right)$ has been used, with $\sigma_{\text {coh, }}=30 \mu \mathrm{m}$ and $\sigma_{\text {coh,y }}=90 \mu \mathrm{m}$. A flat particle form factor $S(q)$ and response function $H(q)$ have been assumed for simplicity.

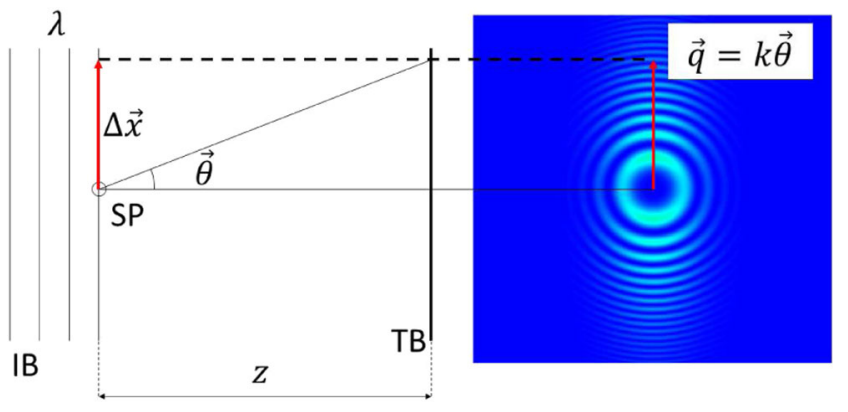

FIG. 4. Geometry relating Fourier wave vectors $\vec{q}$ to transverse displacements $\Delta \vec{r}$. IB represents the incoming beam of wavelength $\lambda$, SP the scattering particle and TB the transmitted beam.

transfer function $H(\vec{q})$ can be accurately characterized with the same experimental setup, as we will show later.

Since the Talbot oscillations in power spectra arise from the cosinusoidal term in the single-particle interferogram, they ultimately describe the effects of the superposition of the scattered and transmitted waves at an angle $\vec{\theta}=\Delta \vec{x} / z$, as presented in Fig. 4. Here $\Delta \vec{x}=(\Delta x, \Delta y)$, as in Eq. (3a).

Standard interferometry and Fourier optics [19] relate the Fourier wave vectors $\vec{q}$ (in reciprocal space) and the angles $\vec{\theta}$ between interfering waves (in direct space) through $\vec{q}=k \vec{\theta}$. This allows to probe spatial coherence over an extended 2-D domain from power spectra of speckle fields, as the Talbot envelope $C(\vec{q})$ actually corresponds to $C(\Delta \vec{x})$, with $\Delta \vec{x}$ given by [20]

$$
\Delta \vec{x}=(\Delta x, \Delta y)=z \frac{\vec{q}}{k} .
$$

The scaling of Eq. (9) introduces the possibility of building one master curve from power spectra at different sample-detector distances, as depicted in Fig. 5. The squared modulus of the 2-D radiation CCF as a function of transverse displacements $\Delta \vec{x}$ can thus be obtained by merging the envelopes of the Talbot oscillations at different $z$ since, according to Eq. (9), higher sample-detector distances allow to access higher transverse displacements, and viceversa. Furthermore, a precise calibration of the detector transfer function can be obtained by acquiring speckle fields very close to the colloidal suspension [30]. In fact, after shot noise and particle form factor reduction, the envelope of the Talbot oscillations is given by $C(\vec{q}) H(\vec{q})$. Then we exploit the fact that for very small sample-detector distances $\Delta \vec{x} \approx \overrightarrow{0}$ according to Eq. (9), hence $C(\overrightarrow{0}) \approx 1$. This procedure is fundamental for X-ray applications due to the anysotropic emission of the phosphors typically used for light conversion, causing a highly q-dependent transfer function.

\section{B. Temporal coherence}

The radiation emitted by a single electron moving along any trajectory is fully temporally coherent regardless of the 

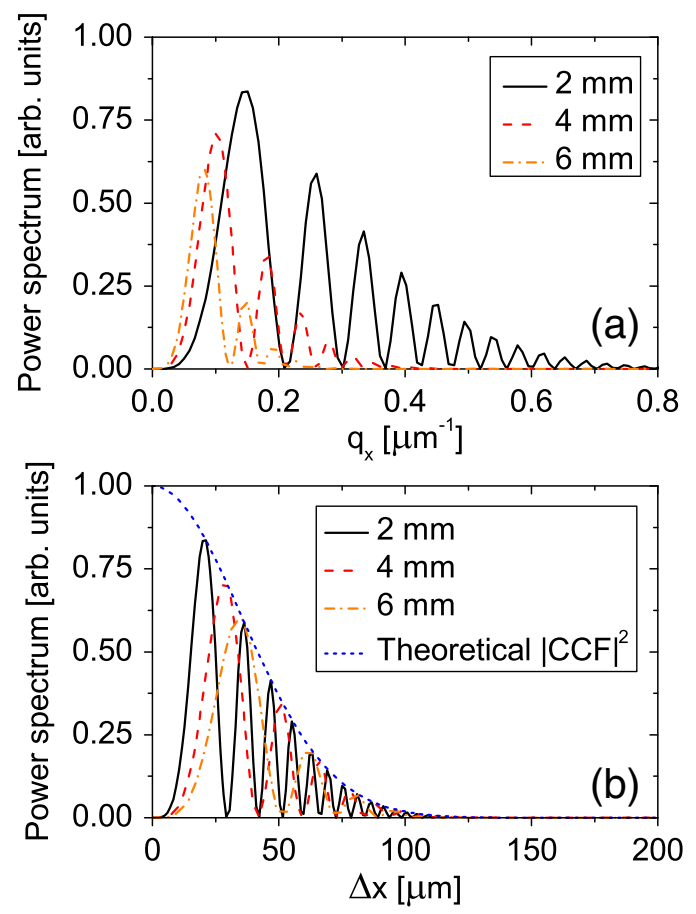

FIG. 5. (a) Simulated horizontal profiles of power spectra for different sample-detector distances. (b) Master curve for spatial coherence obtained through the scaling described in Eq. (9). The common envelope of the reduced power spectra fits the theoretical squared modulus of the radiation $\mathrm{CCF}$ of the form $\exp \left(-\Delta x^{2} / \sigma_{\text {coh }}^{2}\right)$. Parameters used in the simulation are $\lambda=$ $440 \mathrm{~nm}$ and $\sigma_{\mathrm{coh}}=50 \mu \mathrm{m}$.

frequency content of the electric field. This is due to the lack of any stochastic process behind the emission from a single particle, therefore making the field at any time perfectly correlated with the field at any other time. However, a loss of longitudinal coherence might be observed when dealing with electron beams of finite duration within which the electrons are distributed at random times due to shot noise, with such random distribution varying shot by shot. If the observed wavelengths are shorter than the bunch duration, the fields emitted by different electrons add up with random phases and temporal coherence is reduced. Opposite to this case, full temporal coherence would be observed for wavelengths longer than the bunch length as all the emitted wavelets would be endowed with the same phase [26,31]. It follows that to make a statement on coherence, one should compare the single-electron bandwidth with the inverse bunch duration of the electron beam. If the former is much larger than the latter, the stochastic process can be model as (quasi-) stationary and the Wiener-Khinchine theorem can be applied, relating temporal coherence properties of SR along the beamline to the SR spectral density.

Under these assumptions and referring to Fig. 6, the temporal delays $\tau$ between the wavefronts of the scattered spherical waves and the transmitted radiation beam may be

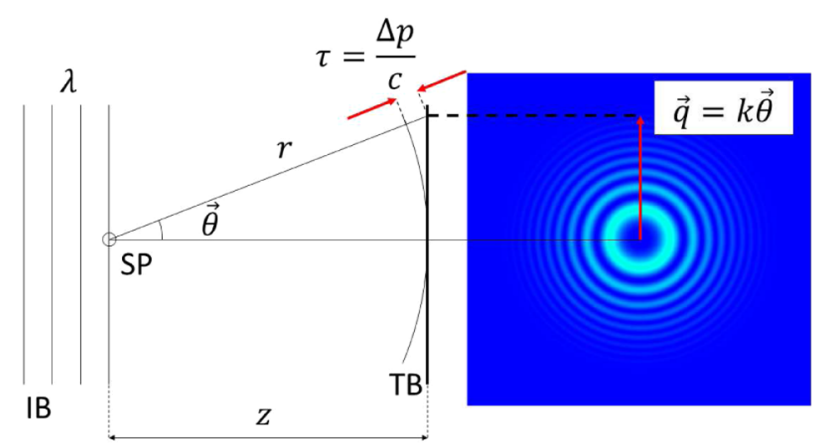

FIG. 6. Geometry relating Fourier wave vectors $\vec{q}$ to the temporal delays $\tau$ associated to the optical path difference $\Delta p$. As explained in the text, only $q=|\vec{q}|$ is relevant, due to circular symmetry in power spectra dominated by temporal coherence. IB represents the incoming beam of wavelength $\lambda$, SP the scattering particle and TB the transmitted beam.

comparable to, or larger than, the radiation inverse bandwidth. In this case, it is useful to work with the average wavelength $\bar{\lambda}$ and $\bar{k}=2 \pi / \bar{\lambda}$. In the rest of the paper we will indicate them with $\lambda$ and $k$, respectively, for the sake of simplicity. Furthermore, the CCF $\mu\left(\Delta x_{i}, \Delta y_{i}\right)$ shall be replaced by the normalized mutual coherence function [MCF or $\Gamma(\Delta x, \Delta y, \tau)]$ of the radiation [27]

$\Gamma(\Delta x, \Delta y, \tau)=\frac{\left\langle E(x, y, t) E^{*}(x+\Delta x, y+\Delta y, t+\tau)\right\rangle}{\sqrt{I_{1}(0) I_{2}(0)}}$

$I_{1}(0)=\left\langle E(x, y, t) E^{*}(x, y, t)\right\rangle$

$I_{2}(0)=\left\langle E(x+\Delta x, y+\Delta y, t) E^{*}(x+\Delta x, y+\Delta y, t)\right\rangle$

which accounts for both spatial and temporal coherence.

For coherence areas large enough, $\Gamma(\Delta x, \Delta y, \tau)=$ $\Gamma(0,0, \tau)$ and the power spectra of speckle fields carry the information on the complex degree of self coherence [CDC or $\gamma(\tau)$ ]

$$
\gamma(\tau)=\frac{\left\langle E(t) E^{*}(t+\tau)\right\rangle}{\left\langle E(t) E^{*}(t)\right\rangle}
$$

which describes the effect of temporal coherence only.

Due to the spherical wavefront of the wave scattered by one particle, temporal delays are the same regardless of the azymuthal angle $\phi$. Therefore, 2-D power spectra exhibit circular symmetry, in contrast to the case of dominant spatial coherence (compare, e.g., Fig. 4 and Fig. 6).

Similarly to narrowband light, Fourier wave vectors can be related to scattering angles, which in turn correspond to optical path differences between the spherical and the transmitted wavefronts, according to Fig. 6: 


$$
\Delta p=r-z=r(1-\cos \theta) \approx z \frac{\theta^{2}}{2}
$$

where $\theta=|\vec{\theta}|$ due to the circular symmetry previously described. Dividing by the speed of light $c$ and substituting $\theta=q / k$ where $q=|\vec{q}|$, the squared modulus of the radiation $\mathrm{CDC}$ is then retrieved with high accuracy from the envelope of Talbot oscillations as a function of temporal delays through the following scaling:

$$
\tau=\frac{z q^{2}}{2 c k^{2}} \text {. }
$$

This scaling of the time delays immediately brings to the possibility to build another master curve where the Talbot oscillations superimpose, as depicted in Fig. 7. This is another peculiar feature of power spectra where the effects of the temporal coherence are dominant, in contrast to the case of spatial coherence treated above.

Dealing with polychromatic light, temporal coherence is influenced by any change in the incoming power spectral density (PSD), for which we need to account the effects of the system transmission. Since the system will generally act as a band-pass filter, these effects can be modeled as the
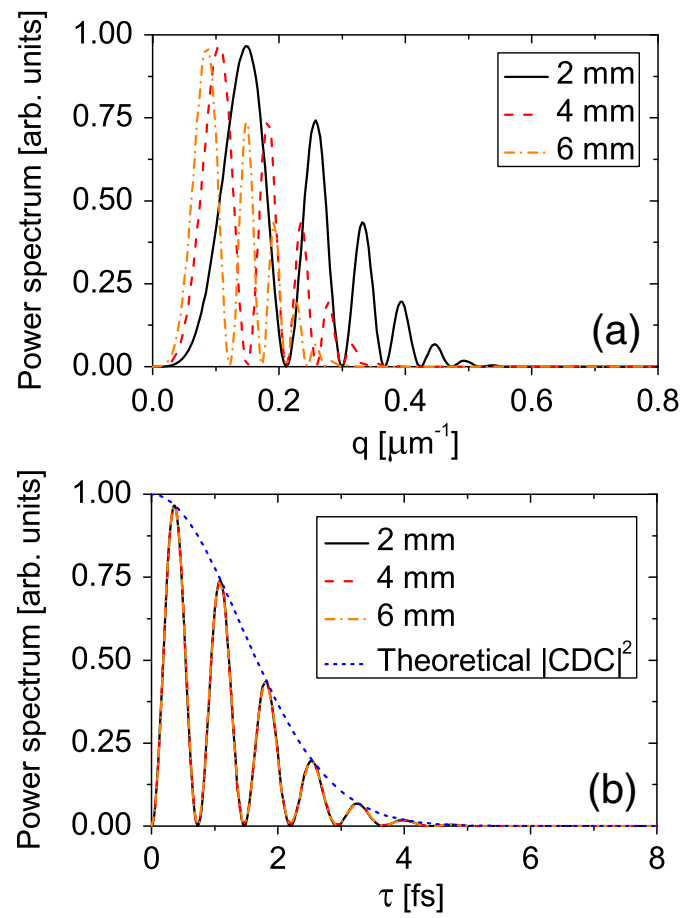

FIG. 7. (a) Simulated radial profiles of power spectra for different sample-detector distances. Here $q=|\vec{q}|$. (b) Master curve for temporal coherence obtained through the scaling described in Eq. (13). In contrast to Fig. 5, Talbot oscillations superimpose. The common envelope of the reduced power spectra fits the theoretical squared modulus of the radiation CDC of the form $\exp \left(-\tau^{2} / t_{\mathrm{coh}}^{2}\right)$. Parameters used in the simulation are $\lambda=440 \mathrm{~nm}$ and $t_{\mathrm{coh}}=2 \mathrm{fs}$. multiplication of the radiation spectrum by the spectral calibration function (SCF). In the HNFS technique, for each wavelength it is given by the product of the scattering amplitude, the transmissivity of the liquid of the suspension and the walls of the cuvette and the spectral response of the CCD, as shown in Fig. 8(a) (each curve has been normalized to a maximum value 1$)$.

The term $|S(0)|$ can be computed from the exact Mie theory of light scattering [29], since spherical nanoparticles are used. Transmission of the liquid and the scattering cell can be measured by means of a standard spectrometer, while the quantum efficiency of the sensor is provided by the CCD constructor. For the experiments at the ALBA bending dipole, the effects of the SCF on the FWHM of the radiation spectrum are negligible, as can be seen in Fig. 8(b). Since the coherence time is of the order of the inverse bandwidth of the radiation [27], we can conclude that the decay of Talbot oscillation is actually a significant measure of the temporal coherence of SR, without the need to correct for the effects of the SCF.
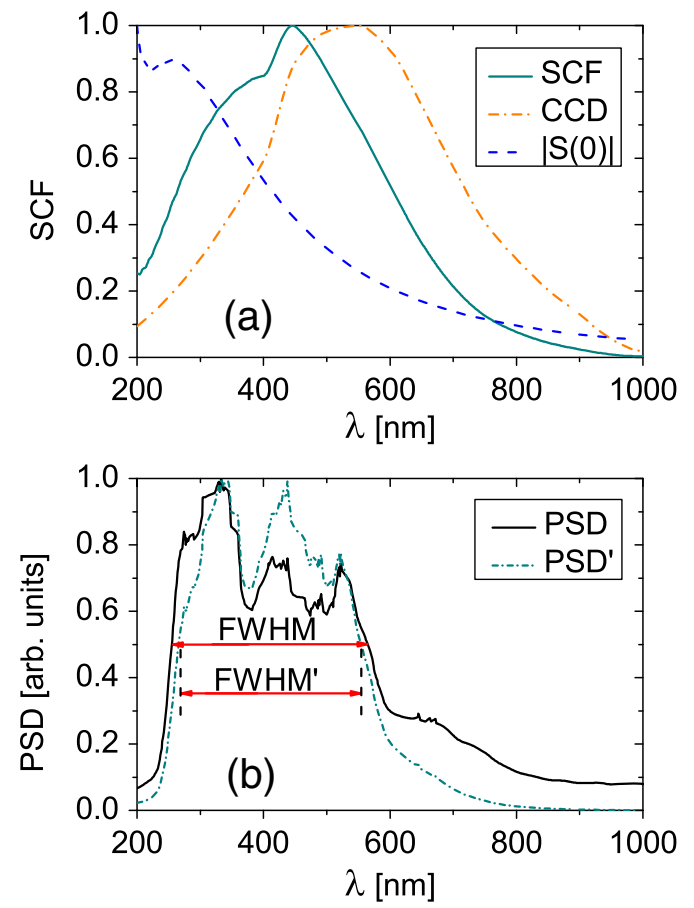

FIG. 8. (a) Spectral calibration function SCF (solid cyan curve) taking into account the dependence of the forward scattering amplitude $|S(0)|$ on wavelength (dashed blue curve) and the quantum efficiency of the CCD sensor (dash-dot orange curve). The measured transmissivity of the liquid in the cuvette and of the cell walls is almost constant over the wavelength range indicated and it is not displayed. (b) Comparison between the incoming power spectral density of SR radiation measured with a spectrometer [32] (PSD, solid black curve) and the detected spectrum (PSD', dash-dot cyan curve) obtained by means of multiplication for the SCF. Its effects on the radiation bandwidth are negligible, since the two spectra have full width at half maximum $F W H M=$ $310 \mathrm{~nm}$ and $F W H M^{\prime}=290 \mathrm{~nm}$, respectively. 


\section{ALBA EXPERIMENTAL SETUP AND DATA PROCESSING}

The method was tested at the ALBA third-generation synchrotron light source. Electrons from a $100 \mathrm{MeV}$ Linac are accelerated to the final energy of $3 \mathrm{GeV}$ by a Booster hosted in the same tunnel as the storage ring. SR generated at Bending Magnet 1 (BM1, radius of curvature $\rho=7.05 \mathrm{~m})$ is extracted through an in-vacuum mirror located at $8.635 \mathrm{~m}$ downstream the source point. The mirror can be inserted from $25 \mathrm{~mm}$ down to $7 \mathrm{~mm}$ from the orbit plane, in order to intercept the emitted visible radiation and not to interfere with the X-rays. A system of 7 flat mirrors transports the selected radiation to the Xanadu beamline where the HNFS diagnostics has been installed, at a distance from the source plane of approximately $15 \mathrm{~m}$. It consists of a quartz cuvette with a colloidal suspension of spherical particles $1 \mu \mathrm{m}$ in diameter. Dilution down to a volume fraction of approximately $10^{-5} \mathrm{w} / \mathrm{w}$ guarantees heterodyne conditions. The speckle fields are collected by a $4 \mathrm{X}$ magnifying microscope objective and imaged onto the sensor of a Basler scA1300-32 cm/gc CCD camera (pixel size is $3.75 \mu \mathrm{m}$, with $1296 \times 966$ pixels). The sampledetector distance $z$ can be changed through a micrometric translation stage and it ranges between $1 \mathrm{~mm}$ and $30 \mathrm{~mm}$. The experimental setup is depicted in Fig. 9.

The single-electron SR spectrum has been calculated starting from the well-known expression of the angularspectral power density [31] and it is reported in Fig. 10 for a point-like aperture positioned at the average vertical

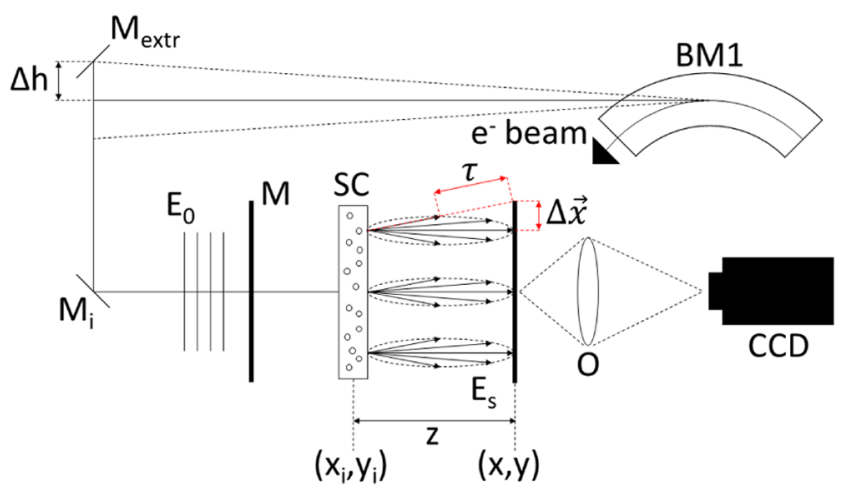

FIG. 9. HNFS experimental setup at ALBA: SR is emitted at Bending Magnet 1 (BM1) and visible light is selected by an extraction mirror $M_{\text {extr }}$ that can be inserted from $25 \mathrm{~mm}$ to $7 \mathrm{~mm}$ from the orbit plane $(\Delta h)$. A system of flat mirrors $\mathrm{M}_{i}$ delivers the radiation $\left(\mathrm{E}_{0}\right)$ to the scattering cell $\mathrm{SC}$ and the interference with the scattered field $\mathrm{E}_{s}$ is imaged by the microscope objective $\mathrm{O}$ onto the CCD. A micrometric translation stage allows to scan the distance $z$ from the particle plane $\left(\mathrm{x}_{i}, \mathrm{y}_{i}\right)$ to the image plane $(\mathrm{x}, \mathrm{y})$. Whether the monochromator $\mathrm{M}$ is used defines narrow-band or broad-band conditions, respectively. The dashed red lines allow to relate the parameter $\Delta x$ of the radiation $\mathrm{CCF}$ and the parameter $\tau$ of the radiation CDC to the geometry of the HNFS setup, as also previously explained in Sec. II A and Sec. II B, respectively.

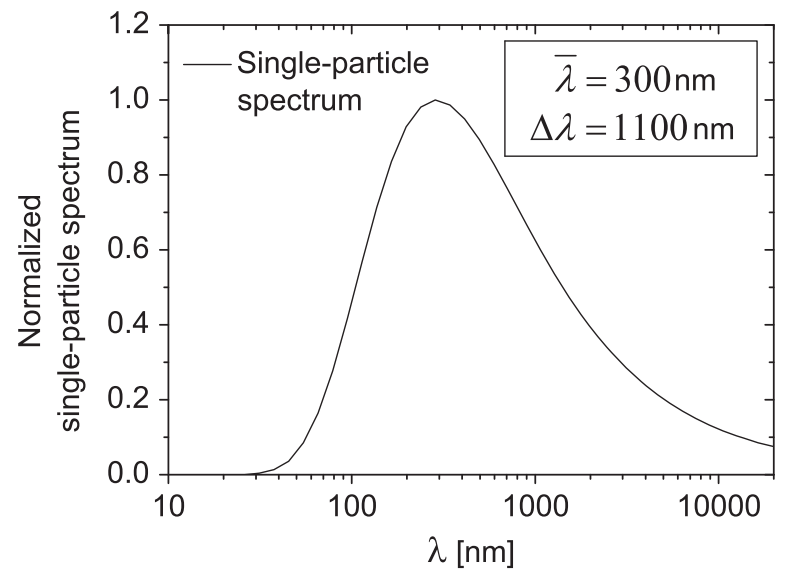

FIG. 10. Computed single-electron spectrum for the ALBA case (radius of curvature $\rho=7.05 \mathrm{~m}$ and Lorentz factor $\gamma=6000)$ at the vertical angle $\psi_{0}=1.835 \mathrm{mrad}$ corresponding to the average vertical position of the in-vacuum extraction mirror. The inverse bandwidth $1 / \Delta \nu_{\mathrm{FWHM}}=\bar{\lambda}^{2} /\left(c \Delta \lambda_{\mathrm{FWHM}}\right)=$ $0.33 \mathrm{fs}$ is much shorter than the bunch duration $\tau_{\text {bunch }}=16 \mathrm{ps}$. This ensures the validity of the Wiener-Khinchine theorem and temporal coherence properties are thus determined by the incident SR spectrum.

angle $\psi_{0}=(16 \mathrm{~mm}) /(8.635 \mathrm{~m})=1.853 \mathrm{mrad}$ of the invacuum mirror. The inverse bandwidth at FWHM is $1 / \Delta \nu_{\mathrm{FWHM}}=0.33 \mathrm{fs}$, much smaller than the bunch duration $\tau_{\text {bunch }}=16 \mathrm{ps}$. When considering a realistic electron beam, this implies that the associated stochastic process is (quasi) stationary and that the SR temporal coherence at any position along the beamline is determined by the SR spectrum by virtue of the Wiener-Khinchine theorem, as discussed in Sec. II B. The actual SR spectrum is affected by the finite solid angle acceptance of the extraction system, by the finite emittance of the particle beam and by the elements of the extraction line (mirrors are out of plane one with respect to the other and their reflectivity depends on the incident wavelength and polarization, and the extraction window further acts as a spectral filter). This results in the actual detected spectrum plotted in Fig. 8. It is worth noting how the measured peak wavelength $(350 \mathrm{~nm})$ is in agreement with the expected one $(300 \mathrm{~nm})$.

For each distance $z$ a stack of 100 images $I_{i}$ separated by a $1 \mathrm{~s}$ time lag is acquired. A square area of $900 \times 900$ pixels is selected from each image and pre-processed by subtracting the dark noise $i_{\text {dark }}$ and by normalizing to the average value $\langle\cdot\rangle_{\text {pixels }}[20]$ :

$$
i_{i}=\frac{I_{i}-i_{\mathrm{dark}}}{\left\langle I_{i}-i_{\mathrm{dark}}\right\rangle_{\mathrm{pixels}}} .
$$

The speckle signal $i_{\mathrm{s}, \mathrm{j}}$ is then obtained by removing the static stray light by means of the double frame analysis [23]: 


$$
i_{\mathrm{s}, \mathrm{j}}=\frac{i_{\mathrm{j}_{1}}-i_{\mathrm{j}_{2}}}{i_{\mathrm{j}_{1}}+i_{\mathrm{j}_{2}}}
$$

where $i_{\mathrm{j}_{1}}$ and $i_{\mathrm{j}_{2}}$ are two independent images with a total temporal separation of at least $50 \mathrm{~s}$. The average power spectrum

$$
I(q)=\sum_{j}\left|\mathcal{F}\left\{i_{\mathrm{s}, \mathrm{j}}\right\}\right|^{2},
$$

with $\mathcal{F}$ denoting the 2-D Fourier transform, is then reduced according to Eq. (8).

\section{RESULTS USING NARROWBAND BEAMS}

Accordingly to the Van Cittert-Zernike theorem, coherence areas generated by an electron beam with rms horizontal size $\sigma_{\mathrm{rms}, \mathrm{x}}=50 \mu \mathrm{m}$ emitting radiation at $440 \mathrm{~nm}$ are as large as $\sigma_{\mathrm{coh}, \mathrm{x}}=\lambda z_{s} /\left(\pi \sigma_{\mathrm{rms}, \mathrm{x}}\right)=4 \mathrm{~cm}$ at a distance from the source plane $z_{s}=15 \mathrm{~m}$, in agreement with independent SR interferometry (SRI) experiments [32]. This value exceeds by more than one order of magnitude the accessible range of transverse displacements $\Delta x_{\max }=2 \mathrm{~mm}$ given by Eq. (9) for a typycal value of $q_{\max }=1 \mu \mathrm{m}^{-1}$ at a sample-detector distance $z=3 \mathrm{~cm}$. The approximation $\Gamma(\Delta x, \Delta y, \tau)=\Gamma(0,0, \tau)$ made in Sec. II B is valid and it allows to measure temporal coherence effects even for narrowband beams. By contrast, the SR spatial coherence would be easily accessed at shorter x-ray wavelengths, because the generated coherence areas would then be much smaller [18,21].

Narrowband conditions are ensured by a band pass filter with FWHM bandwidth of $10 \mathrm{~nm}$ by Thorlabs, centered at an average wavelength of $440 \mathrm{~nm}$ [33]. Figure 11 shows the resulting 2 dimensional power spectra. Perfect azimuthal symmetry of the Talbot oscillations is the signature of limiting temporal coherence.

This is further confirmed in Fig. 12, which shows how power spectra for different distances $z$ superimpose in one master curve under the scaling law of Eq. (13). The

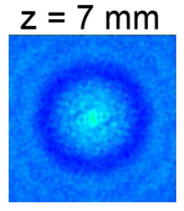

$z=15 \mathrm{~mm}$

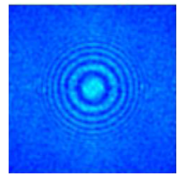

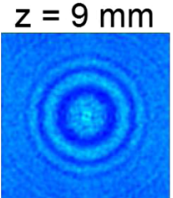

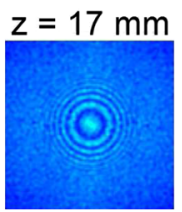

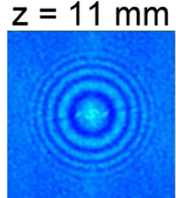
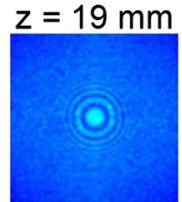
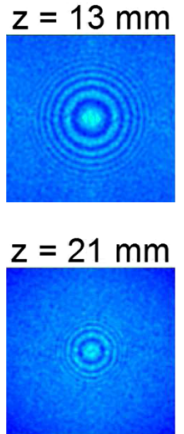

FIG. 11. Raw power spectra in narrowband conditions. Perfect azymuthal symmetry is a clear signature of predominant temporal coherence, as discussed in Sec. II B.

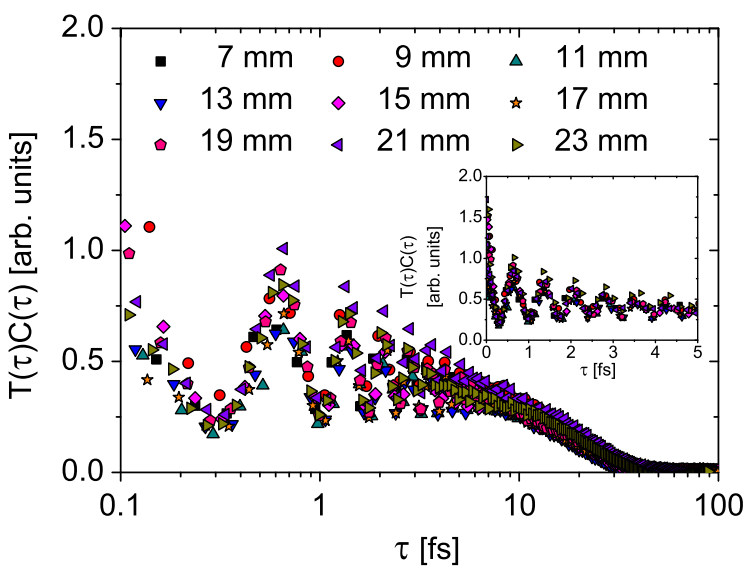

FIG. 12. Power spectra fit the master curve under the scaling of Eq. (13). Inset shows the enlarged region corresponding to $\tau<5$ fs to evidence the fine superposition of Talbot oscillations.

good fit to the temporal master curve proves full spatial coherence over the accessible transverse displacements of roughly $1 \mathrm{~mm}$.

The finite thickness of the sample and the walkoff effect related to the limited sensor size [34-36] induce a tapering on Talbot oscillations clearly visible for $\tau<10 \mathrm{fs}$. This prevents to generate the master curves upon the scalings described in Eq. (9) or Eq. (13) from the term $C(q) T(q)$, unless the average of the upper and lower envelopes is computed, as shown in Fig. 13.

The measured squared modulus of the radiation CDC is related to a coherence time

$\tau_{\mathrm{coh}}=\int_{-\infty}^{+\infty}|\gamma(\tau)|^{2} d \tau=2 \int_{0}^{+\infty} C(\tau) d \tau=40 \pm 10 \mathrm{fs}$,

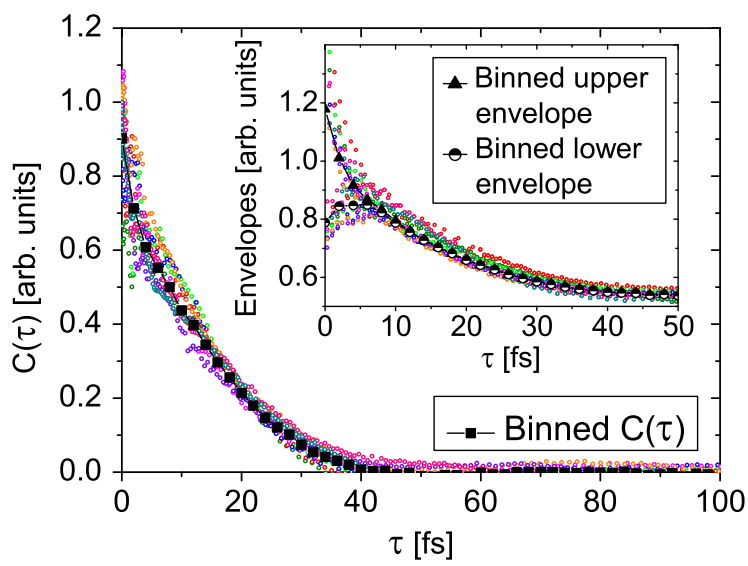

FIG. 13. Squared modulus of the radiation CDC as measured with the HNFS technique. Data from the different 9 sampledetector distances shown in Fig. 12 have been merged through Eq. (13) and binned. Inset shows the corresponding upper and lower envelopes. The tapering effect of the modified Talbot transfer function is clearly visible for $\tau<10 \mathrm{fs}$. 
in good agreement with the value given by the filter inverse bandwidth $\tau_{\mathrm{coh}}^{\text {filt }}=0.664 \lambda^{2} /(c \Delta \lambda)=43 \mathrm{fs}$, where the multiplicative factor 0.664 takes into account the Gaussian-like line shape of the filter $[27,28]$. The error bar stems from the dispersion of data around the binned curve.

Even if the large wavelength prevents full measurements of SR spatial coherence, useful information can be obtained by analyzing narrowband Talbot oscillations. In fact, Fourier optics predicts that coherence areas should carry the information on the wavefront curvature for visible synchrotron radiation. Since the transition from the near field to the far field of the electron beam occurs at $z_{\mathrm{FF}}=\sigma_{\mathrm{rms}}^{2} / \lambda=7 \mathrm{~mm}$, the sample lies in the Fraunhofer zone of the source $\left(z_{s}=15 \mathrm{~m} \gg z_{\mathrm{FF}}\right)$ where coherence patches are homothetic with the same curvature of the wavefront. For SR wavefronts with radius of curvature $R$, the Talbot oscillations at a distance $z$ from the colloids are described in terms of an effective sample-detector distance $z_{\text {eff }}$ given by

$$
\frac{1}{z_{\mathrm{eff}}}=\frac{1}{R}-\frac{1}{z}
$$

Since this relation is nonlinear, the wavefront curvature can be measured by comparing $z_{\text {eff }}$ (fitted from the position of Talbot maxima and minima) to the nominal $z$ (known by the position of the translation stage). Results are reported in Fig. 14 and the fitted value for the radius of curvature according to Eq. (18) is $R \approx 15 \mathrm{~m}$, corresponding to the distance of the scattering cell from the center of the bending dipole. However, due to the low signal caused by the monochromator, data are widely scattered and affected by large uncertainties. As a result, we can only conservatively state that data are compatible with a radius of curvature larger than $5 \mathrm{~m}$.

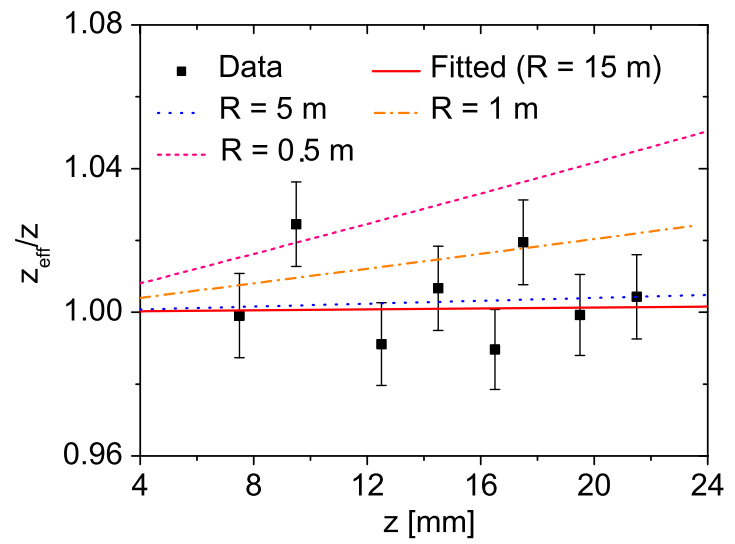

FIG. 14. The periodicity of the Talbot oscillations is affected by a non-vanishing curvature of the coherence areas. Since for visible wavelength the coherence areas at the sample position behave as far field speckles, they carry the same curvature as the SR wavefront. Data are compatible with a radius of curvature larger than $5 \mathrm{~m}$.
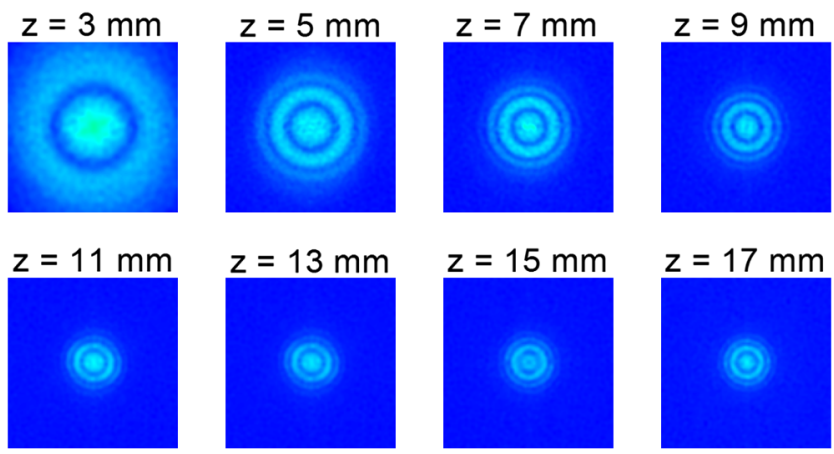

$z=17 \mathrm{~mm}$

FIG. 15. Raw power spectra of broadband synchrotron radiation. Perfect azymuthal symmetry is a clear signature of predominant temporal coherence, as discussed in Sec. II B.

\section{RESULTS USING WHITE BEAMS}

Figure 15 shows the 2-D power spectra obtained using the experimental setup in Fig. 9, but as opposed to the case in Fig. 11, no band-pass filter is used.

The master curve from power spectra at different distances is shown in Fig. 16 and it displays less Talbot oscillations than in Fig. 12, implying a shorter coherence length.

This is also evident in the retrieved squared modulus of the radiation CDC shown in Fig. 17, where data are much less scattered than in Fig. 13 due to the high signal level in the CCD camera without any monochromator. Integration of the master curve $C(\tau)$ [as in Eq. (17)] yields a coherence time of $1.6 \pm 0.4 \mathrm{fs}$, corresponding to a FWHM bandwidth of the power spectral density $\Delta \lambda=240 \mathrm{~nm}$ at an average wavelength $\bar{\lambda}=350 \mathrm{~nm}$. This value of the coherence time is compatible within the experimental error with the inverse bandwidth of $1.4 \mathrm{fs}$ obtained from the spectrometer measurements.

Since we can only access the modulus of the radiation CDC, we cannot directly apply the Wiener-Kintchine

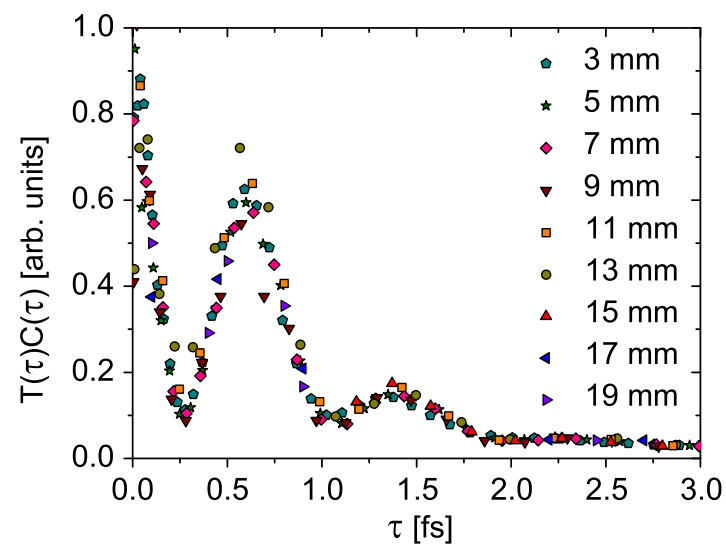

FIG. 16. Broadband power spectra fit the master curve under the scaling of Eq. (13). The 9 probed distance are indicated in the figure legend and range between $3 \mathrm{~mm}$ and $19 \mathrm{~mm}$. 


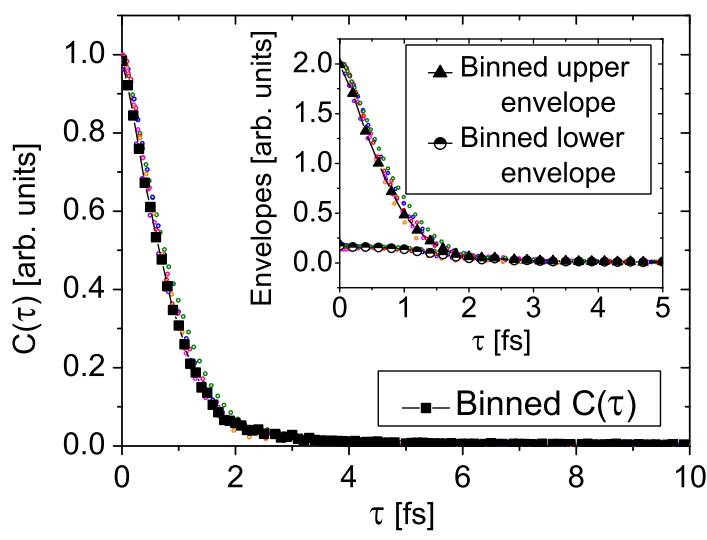

FIG. 17. Squared modulus of the radiation CDC as measured with the HNFS technique. Data from the 9 different sampledetector distances shown in Fig. 16 have been merged through Eq. (13) and binned. Inset shows the corresponding upper and lower envelopes. By contrast with Fig. 13, the short coherence time of the radiation makes any tapering effect negligible, as the loss of Talbot visibility and the decay of power spectra to shot noise level are entirely due to the radiation CDC.

theorem to retrieve the SR spectrum at the sample position. However, assuming that the SR spectrum is roughly symmetric around the peak wavelength,

$$
\gamma(\tau)=|\gamma(\tau)| e^{i \bar{\nu} \tau}
$$

being $\bar{\nu}=c / \bar{\lambda}$ the average frequency, and the WienerKintchine theorem takes the modified form [37]

$$
S(\nu)=\int_{0}^{+\infty} 2|\gamma(\tau)| \cos [(\nu-\bar{\nu}) \tau] d \tau
$$

Figure 18 shows the power spectral density (PSD) obtained by applying Eq. (20) to the measured curve $C(\tau)$ of Fig. 17. Comparison with independent measurements (red line in Fig. 18) performed with a standard spectrometer shows how the overall shape of the SR spectrum at the sample position is satisfactorily reproduced. The three narrow peaks are missing in the retrieved spectrum mainly due to shot noise limitations. In fact, since their width is less than one half of the spectrum FWHM, they are expected to contribute the modulus of the radiation CDC with some echoes at temporal delays larger than $\tau \approx 2 \tau_{\text {coh }}=3.4 \mathrm{fs}$, where the decay of Talbot oscillations has already reached the shot noise level, as can be seen in Fig. 16, and where sampling is not properly done due to the quadratic relation of Eq. (13). Moreover, referring to Fig. 16, we also stress that $C(\tau)$ is computed by interpolation on a small number of Talbot maxima and minima and that we assumed specific symmetry properties for the SR spectrum in order to write Eq. (20) which are rigorously valid, in our case, only close to the peak wavelength. Although this approach allows to correctly retrieve larger

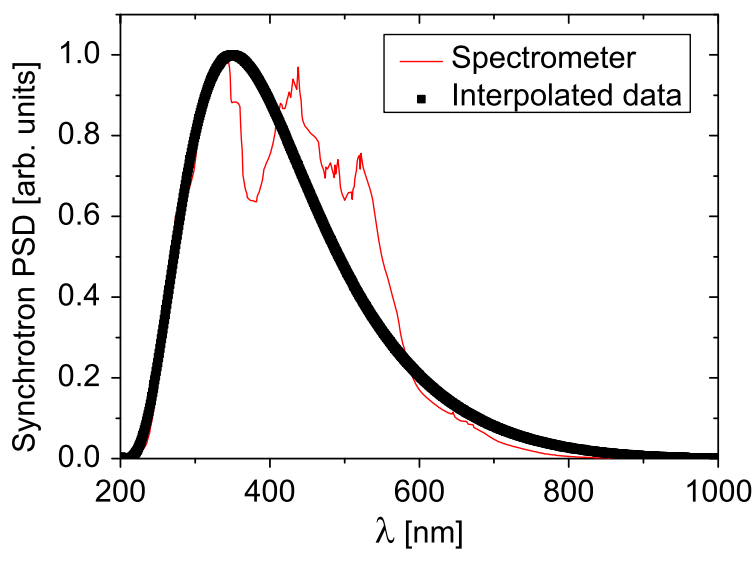

FIG. 18. Synchrotron power spectral density PSD retrieved with the HNFS technique (squares) and measured with a spectrometer (red solid curve). The overall shape of the spectrum has been satisfactorily reproduced, despite the three narrow peaks have not been retrieved (see text for details).

structures of the SR power spectral density, it is currently a limiting factor for the detection of finer details.

The comparison with the results from the spectrometer has been used as a benchmark of the technique, which is capable of retrieving the overall shape of the incident PSD though finer details are missed. Nonetheless, the measured FWHM spectral bandwidth, conveying the information on the SR coherence time, is correctly retrieved $(250 \mathrm{~nm}$ to be compared with $\sim 300 \mathrm{~nm}$ from spectrometry measurements). Despite in the visible range there certainly are more accurate methods to characterize the SR spectrum and temporal coherence, we would like to recall the advantages of the HNFS approach over other techniques: it is wavelength independent, the setup is compact and it does not require any dedicated optics, thus being suitable to be operated at x-ray wavelengths; it is free of severe alignment constraints; and it allows to investigate spatial and temporal coherence by exploiting the same experimental setup.

\section{CONCLUSIONS}

We have developed a diagnostics technique based on heterodyne near field speckles for the characterization of both spatial and temporal coherence properties of visible synchrotron radiation. The method relies on the possibility of building master curves under proper scaling laws for the spatial and the temporal coherence. The experimental setup is minimal and easy to implement and data processing is model independent and free of any external parameter.

We have performed for the first time measurements of temporal coherence effects in synchrotron radiation with both narrowband and white beams. In the former case, a coherence time of $40 \pm 10$ fs has been measured, compatible with the filter inverse linewidth (expected value $43 \mathrm{fs}$ ). The high quality of the master curve proves full spatial coherence over the accessed transverse scale of $1 \mathrm{~mm}$. 
Since for visible wavelengths the sample lies in the far field of the source, coherence patches are homotetic and they carry the information on the wavefront curvature. By studying the periodicity of the Talbot oscillations as the sampledetector distance is varied, we have estimated a wavefront radius of curvature larger than $5 \mathrm{~m}$ (less conservatively, the fitted value is compatible with the nominal distance of $15 \mathrm{~m}$ from the center of the bending dipole). By contrast, it is worth noting that for $\mathrm{x}$-ray wavelengths the transition to the far field of the electron beam occurs after tens or hundreds of meters and the sample would be in the Fresnel zone of the source $\left(z_{s} \ll z_{\mathrm{FF}}\right)$. Near field coherence patches behave as diffraction limited wavefronts with a Rayleigh range of the order of some meters, much larger than the typical sampledetector distances $z$. The scattered spherical waves would thus be heterodyned by a wavefront carrying vanishing curvature.

Regarding white beams, the decay of speckle power spectra yields a coherence time of $1.6 \pm 0.4 \mathrm{fs}$, related to a radiation bandwidth of $240 \mathrm{~nm}$ at an average wavelength of $350 \mathrm{~nm}$. From the measured squared modulus of the radiatoin Complex Degree of Coherence, we have retrieved the synchrotron power spectral density by applying the modified Wiener-Kintchine theorem. Results are in good agreement with independent measurements performed with a spectrometer, showing how HNFS can be effectively implemented as a powerful diagnostics technique.

[1] D. Mills, Third Generation Hard X-ray Synchrotron Radiation Sources (John Wiley and Sons, Inc., New York, 2002).

[2] K. A. Nugent, Coherent methods in the X-ray sciences, Adv. Phys. 59, 1 (2010).

[3] H. Wiedemann, Particle Accelerator Physics (Springer, Berlin, 2007).

[4] T. Naito and T. Mitsuhashi, Very small beam-size measurement by a reflective synchrotron radiation interferometer, Phys. Rev. ST Accel. Beams 9, 122802 (2006).

[5] A. Rousse, K. T. Phouc, R. Shah, A. Pukhov, E. Lefebvre, V. Malka, S. Kiselev, F. Burgy, J.-P. Rousseau, D. Umstadter, and D. Hulin, Production of a keV X-Ray Beam from Synchrotron Radiation in Relativistic Laser-Plasma Interaction, Phys. Rev. Lett. 93, 135005 (2004).

[6] S. Cipiccia et al., Gamma-rays from harmonically resonant betatron oscillations in a plasma wake, Nat. Phys. 7, 867 (2011).

[7] A. Curcio, M. Anania, F. Bisesto, E. Chiadroni, A. Cianchi, M. Ferrario, F. Filippi, D. Giulietti, A. Marocchino, M. Petrarca, V. Shpakov, and A. Zigler, Trace-space reconstruction of low-emittance electron beams through betatron radiation in laser-plasma accelerators, Phys. Rev. Accel. Beams 20, 012801 (2017).

[8] B. Paroli, E. Chiadroni, M. Ferrario, V. Petrillo, M. A. C. Potenza, A. R. Rossi, L. Serafini, and V. Shpakov, Asymmetric lateral coherence of betatron radiation emitted in laser-driven light sources, Europhys. Lett. 111, 44003 (2015).

[9] D. Paterson, B. E. Allman, P. J. McMahon, J. Lin, N. Moldovan, K. A. Nugent, I. McNulty, C. T. Chantler, C. C. Retsch, T. H. K. Irving, and D. C. Mancini, Spatial coherence measurement of X-ray undulator radiation, Opt. Commun. 195, 79 (2001).

[10] V. Kohn, I. Snigireva, and A. Snigirev, Direct Measurement of Transverse Coherence Length of Hard X-rays from Interference Fringes, Phys. Rev. Lett. 85, 2745 (2000).

[11] M. Masaki and S. Takano, Two-dimensional visible synchrotron light interferometry for transverse beam-profile measurement at the SPring-8 storage ring, J. Synchrotron Radiat. 10, 295 (2003).

[12] J. J. A. Lin, D. Paterson, A. G. Peele, P. J. McMahon, C. T. Chantler, K. A. Nugent, B. Lai, N. Moldovan, Z. Cai, D. C. Mancini, and I. McNulty, Measurement of the Spatial Coherence Function of Undulator Radiation using a Phase Mask, Phys. Rev. Lett. 90, 074801 (2003).

[13] F. Pfeiffer, O. Bunk, C. Schulze-Briese, A. Diaz, T. Weitkamp, C. David, J. F. van der Veen, I. Vartanyants, and I. K. Robinson, Shearing Interferometer for Quantifying the Coherence of Hard X-Ray Beams, Phys. Rev. Lett. 94, 164801 (2005).

[14] X. Shi, S. Marathe, M. J. Wojcik, N. G. Kujala, A. T. Macrander, and L. Assoufid, Circular grating interferometer for mapping transverse coherence area of Xray beams, Appl. Phys. Lett. 105, 041116 (2014).

[15] M. Lyubomirskiy, I. Snigireva, and A. Snigirev, Lens coupled tunable Young's double pinhole system for hard $\mathrm{X}$-ray spatial coherence characterization, Opt. Express 24 13679 (2016).

[16] V. Hilbert, A. Blinne, S. Fuchs, T. Feigl, T. Kampfer, C. Rodel, I. Uschmann, M. Wunsche, G. G. Paulus, E. Forster, and U. Zastrau, An extreme ultraviolet Michelson interferometer for experiments at freeelectron lasers, Rev. Sci. Instrum. 84, 095111 (2013).

[17] J. P. Sutter, T. Ishikawa, U. Kuetgens, G. Materlik, Y. Nishino, A. Rostomyan, K. Tamasaku, and M. Yabashi, An X-ray BBB Michelson interferometer, J. Synchrotron Radiat. 11, 378 (2004).

[18] M. D. Alaimo, M. A. C. Potenza, M. Manfredda, G. Geloni, M. Sztucki, T. Narayanan, and M. Giglio, Probing the Transverse Coherence of an Undulator X-ray Beam Using Brownian Particles, Phys. Rev. Lett. 103, 194805 (2009).

[19] J. W. Goodman, Introduction to Fourier Optics (Roberts and Company Publishers, New York, 2004).

[20] M. D. Alaimo et al., Mapping the transverse coherence of the self amplified spontaneous emission of a free-electron laser with the heterodyne speckle method, Opt. Express 22, 30013 (2014).

[21] Y. Kashyap, H. Wang, and K. Sawhney, Two-dimensional transverse coherence measurement of hard-X-ray beams using near-field speckle, Phys. Rev. A 92, 033842 (2015).

[22] M. Siano, B. Paroli, E. Chiadroni, M. Ferrario, and M. A. C. Potenza, Measurement of power spectral density of broad-spectrum visible light with heterodyne near field scattering and its scalability to betatron radiation, Opt. Express 2332888 (2015). 
[23] F. Ferri, D. Magatti, D. Pescini, M. A. C. Potenza, and M. Giglio, Heterodyne near-field scattering: A technique for complex fluids, Phys. Rev. E 70, 041405 (2004).

[24] D. Magatti, M. D. Alaimo, M. A. C. Potenza, and F. Ferri, Dynamic heterodyne near field scattering, Appl. Phys. Lett. 92, 241101 (2008).

[25] J. W. Goodman, in Laser Speckle and Related Phenomena (Springer, Berlin, 1975).

[26] G. Geloni, E. Saldin, E. Schneidmiller, and M. Yurkov, Transverse coherence properties of X-ray beams in thirdgeneration synchrotron radiation sources, Nucl. Instrum. Methods Phys. Res., Sect. A 588, 463 (2008).

[27] J. W. Goodman, Statistical Optics (Wiley-Interscience, New York, 2000).

[28] M. Born and E. Wolf, Priciples of Optics (Cambridge University Press, Cambridge, England, 1999).

[29] H. C. van de Hulst, Light Scattering by Small Particles (John Wiley and Sons, New York, 1957).

[30] R. Cerbino, L. Peverini, M. A. C. Potenza, A. Robert, P. Bosecke, and M. Giglio, X-ray scattering information obtained from near-field speckle, Nat. Phys. 4, 238 (2008).
[31] A. Hofmann, The Physics of Synchrotron Radiation (Cambridge University Press, Cambridge, England, 2004).

[32] L. Torino, Ph.D. thesis, Università di Pisa, 2017.

[33] https://www.thorlabs.com/thorproduct.cfm?partnumber= FB440-10.

[34] M. Giglio, D. Brogioli, M. A. C. Potenza, and A. Vailati, Near field scattering, Phys. Chem. Chem. Phys. 6, 1547 (2004).

[35] S. Mazzoni, M. A. C. Potenza, M. D. Alaimo, S. J. Veen, M. Dielissen, E. Leussink, J. L. Dewandel, O. Minster, E. Kufner, G. Wegdam, and P. Schall, SODI-COLLOID: A combination of static and dynamic light scattering on board the International Space Station, Rev. Sci. Instrum. 84, 043704 (2013).

[36] M. A. C. Potenza, K. P. V. Sabareesh, M. Carpineti, M. D. Alaimo, and M. Giglio, How to Measure the Optical Thickness of Scattering Particles from the Phase Delay of Scattered Waves: Application to Turbid Samples, Phys. Rev. Lett. 105, 193901 (2010).

[37] E. Wolf, Introduction to the Theory of Coherence and Polarization of Light (Cambridge University Press, Cambridge, England, 2007). 